\title{
Anesthesia for Neurosurgery (Part I)
}

\author{
Lalit Gupta ${ }^{1}$, Bhavna Gupta,* \\ ${ }^{\mathbf{1}}$ Assistant Professor, ${ }^{2}$ Senior Resident, Dept. of Anaesthesia, Maulana Azad Medical College, New Delhi, Delhi, India
}

*Corresponding Author:

Email: bhavna.kakkar@gmail.com

Received: $09^{\text {th }}$ January, 2018

Accepted: $18^{\text {th }}$ January, 2018

\begin{abstract}
The central nervous system (CNS) deserves special consideration in the perioperative setting for several reasons for an anesthetist. An understanding of neuroanatomy is essential because neuro anesthesia continues to develop and evolution of neurosurgical practice is accompanied by new challenges for the anesthetist. Basic knowledge and expertise of the neuro-anesthetist can directly influence patient outcome. With the recent advancement in functional and minimally invasive procedures, there is an increased emphasis on the provision of optimal operative conditions, preservation of neurocognitive function, minimizing interference with electrophysiological monitoring, and a rapid, high-quality recovery. So, during neuro anesthesia, anesthesiologist needs to know physiology of CNS including cerebral metabolism and cerebral blood flow. Neuro anesthesia can be challenging, because sometimes apparently contradictory demands must be managed, for example, achieving optimal conditions for neurophysiological monitoring while maintaining sufficient anesthetic depth, or maintaining oxygen delivery to neuronal tissue and simultaneously preventing high blood pressures that might induce local bleeding. One of the peculiarities of neuro anesthesia has always been that as much importance is attached to wakening the patient as sending them to sleep.
\end{abstract}

\section{A. Cerebral Anatomy for Anesthetists}

1. The brain and spinal cord are surrounded by protective but non-distensible bony structure called cranium.

2. Cranium is divided into supra-tentorial and infratentorial compartments. The supra-tentorial compartment contains the cerebral hemispheres and diencephalon (thalamus and hypothalamus), whereas the brainstem and cerebellum make up the infra-tentorial compartment.

3. The intracranial volume is fixed, thereby providing little room for anything other than the brain, cerebrospinal fluid (CSF), and blood contained in the cerebral vasculature. It is in the context of the restrictive nature of the space that needs urgent interventions.

4. The anterior cerebral circulation originates from the carotid artery, the posterior circulation results from the vertebral arteries, and the system of collateralization is known as the circle of Willis.

5. The spinal column is the bony structure made up of the seven cervical, 12 thoracic, and five lumbar vertebrae, as well as the sacrum. The spinal cord is a long, thin, tubular bundle of nervous tissue and support cells that extends from the medulla oblongata in the brainstem to the lumbar region of the vertebral column. The brain and spinal cord together make up the central nervous system (CNS)

6. The spinal cord exits the skull through the foramen magnum and enters the canal formed by the vertebral bodies. In adults, the spinal cord typically ends at the lower aspect of the first lumbar vertebral body.
7. The anterior spinal artery arises from the vertebral arteries and supplies the anterior two thirds of the spinal cord. This vessel runs the length of the cord, receiving contribution from radicular arteries via intercostal vessels. The artery of Adamkiewich is the most important radicular artery

8. The posterior third of the cord is supplied by two posterior spinal arteries, which arise from the vertebral arteries and also receive contribution from radicular arteries

9. The term "blood-brain barrier" was coined, by Lewandowsky in 1898. The blood-brain barrier is composed of capillary endothelial cells with tight junctions that prevent free passage of macromolecules or proteins. In contrast, lipidsoluble substances (carbon dioxide, oxygen, anesthetic drugs) cross the blood-brain barrier easily. The blood-brain barrier may be disrupted by acute systemic hypertension, trauma, infection, arterial hypoxemia, severe hypercapnia, tumors, or sustained seizure activity.

\section{B. Neurophysiology}

The brain, although being $2 \%$ of the total body weight $(\sim 1350 \mathrm{gm})$, receives a disproportionately large share of cardiac output. Normal cerebral blood flow (CBF) is approximately $50 \mathrm{ml} / 100 \mathrm{~g} / \mathrm{min}$ and represents $12 \%$ to $15 \%$ of total cardiac output. Despite dependence on such high cardiac output, brain is not an energy storehouse. Nearly $40 \%$ of total cerebral energy utilization is used to maintain the cellular homeostatic activities of brain tissues (i.e. basic functions of cell). While remaining $60 \%$ of the total cerebral energy utilization is needed to maintain the electrophysiological 
function of brain such as depolarization-repolarization, transport and reuptake of neurotransmitter.

At rest for production of energy the human brain consumes $\mathrm{O}_{2}$ at an average rate of $3.5 \mathrm{ml} / 100 \mathrm{gm}$ of brain tissue/min $\left(\mathrm{CMRO}_{2}\right)$. Therefore, the whole human brain consumes $\mathrm{O}_{2}$ at the rate of $47 \mathrm{ml} / \mathrm{min}(13.5 \times 3.5=47)$. This represents $20 \%$ of the total body $\mathrm{O}_{2}$ consumption in a minute.

Normally, the rate of utilization of glucose by brain is $5 / 100 \mathrm{mg}$ of tissue/min. But, during starvation ketone bodies such as $\beta$-hydroxybutyric acetate and acetoacetate and acetone also become the substrate for energy production. In the absence of supply of $\mathrm{O}_{2}$ lactic acid is formed as a result of anaerobic glycolysis with the little production of energy.

\section{Cerebral Blood Flow Regulation}

CBF including gray matter and white matter is estimated to about $675 \mathrm{ml} / \mathrm{min}(13.5 \times 50=675)$. The $\mathrm{CBF}$ has a critical value below which level the function of brain deteriorates. When the CBF has fallen to 20 $\mathrm{ml} / 100 \mathrm{gm}$ of tissue/min then the evidence of ischemia of brain in EEG begins to appear. While at CBF below $10 \mathrm{ml} / 100 \mathrm{gm}$ of tissue/min, the irreversible damage of brain occurs.

Auto regulation of CBF depends on chemical, myogenic, neurogenic and few extrinsic mechanisms.

1. The chemical mechanism for the regulation of $\mathrm{CBF}$ includes the changes in $\mathrm{CMRO}_{2}, \mathrm{PaCO}_{2}$, and $\mathrm{PaO}_{2}$.

i. Within this range of change in $\mathrm{PaCO}_{2}$ (30-70 mm $\mathrm{Hg}$ ) the $\mathrm{CBF}$ changes by 1 to $2 \mathrm{ml} / 100 \mathrm{gm}$ of tissue/min for each $1 \mathrm{~mm}$ of $\mathrm{Hg}$ change in $\mathrm{PaCO}_{2}$ but this response is attenuated when the $\mathrm{PaCO}_{2}$ falls below $25 \mathrm{~mm}$ of $\mathrm{Hg}$.

ii. Changes in $\mathrm{PaO}_{2}$ between 60 to $300 \mathrm{~mm}$ of $\mathrm{Hg}$ have little impudence on CBF directly. But with $\mathrm{PaO}_{2}$ below $60 \mathrm{~mm}$ of $\mathrm{Hg}, \mathrm{CBF}$ increases rapidly due to vasodilatation caused by hypoxia.

2. Myogenic (auto) regulation: Over a wide range of change in mean arterial pressure (MAP) the CBF is maintained automatically by changing the resistance of cerebral vessels (constriction or dilatation) intrinsically. This is called as the myogenic or autoregulation of CBF.

i. The normal range of MAP through which the CBF is maintained by autoregulation at constant level is 60 to $160 \mathrm{~mm}$ of $\mathrm{Hg}$. Above and below this level $\mathrm{CBF}$ is not auto-regulated and becomes pressure dependent

ii. The difference between the MAP and ICP (intracranial pressure) or CVP which will be the greater is known as the cerebral perfusion pressure (CPP), which determines the rate of diffusion of substrates into the brain tissue from vessels. When the MAP goes above $160 \mathrm{~mm}$ of $\mathrm{Hg}$ and auto regulation fails, then ICP rises and CPP falls. Due to any condition when CVP exceeds ICP, then CPP will be the difference between MAP and CVP. Thus the CPP = MAP -ICP or MAP - CVP.

3. Neurogenic mechanism or regulation: the neurogenic regulation exists only on the large cerebral vessels and is primarily due to sympathetic (vasoconstrictive), parasympathetic (vasodilatory), serotonergic innervations. Although the density of innervation decreases with the decrease of vessel's size, still it may play an important role in some pathological states (hemorrhagic shock, trauma, and stroke).

4. Extrinsic mechanisms or factors: it involves temperature, viscosity of blood and external vasoactive drugs.

i. For every $1{ }^{\circ} \mathrm{C}$ reduction of temperature (hypothermia) of brain $\mathrm{CMRO}_{2}$ and $\mathrm{CBF}$ decreases by 5 to $6 \%$. Between $37^{\circ} \mathrm{C}$ to $42^{\circ} \mathrm{C}$ both the $\mathrm{CMRO}_{2}$ and $\mathrm{CBF}$ increase. But above $42^{\circ} \mathrm{C}$ (threshold level) the $\mathrm{CMRO}_{2}$ again begins to fall due to the neuronal cell damage resulting from protein degradation.

ii. With hematocrit value within the normal range (33 to 45) no significant change in CBF occurs but beyond this range the changes in $\mathrm{CBF}$ due to changes in hematocrit value are more substantial. A decrease in hematocrit value (anemia) improves the CBF due to the decrease in viscosity of blood. Optimum delivery of $\mathrm{O}_{2}$ occurs at hematocrit value of $30 \%$. Increased hematocrit and viscosity in polycythemia has opposite effect and reduce CBF.

iii. The systemic vasodilators used for induced hypotension during anesthesia such as nitroglycerine, calcium channel blockers, etc, cause cerebral vasodilatation and increase CBF. If hypotension is induced slowly by these agents, then ICP does not rise because of shifting of CSF and venous blood (as compensatory mechanism) when changes in increase of CBF occur very slowly. Otherwise, vasodilator drugs will cause increased CBF and increased ICP.

iv. The $\alpha 1$ agonists do not increase CBF; though cause acute increase in MAP due to the presence of autoregulation and intact blood brain barrier (BBB). The $\beta$ blockers probably have no effect on $\mathrm{CBF}$ or reduce it with the reduction of $\mathrm{CMRO}_{2}$. Dopamine in low doses $(2-6 \mu \mathrm{g} / \mathrm{Kg} / \mathrm{min})$ probably causes the dilatation of cerebral vessels and increase in CBF. While it causes vasoconstriction and reduction of $\mathrm{CBF}$ in higher doses (6-20 $\mu \mathrm{g} / \mathrm{Kg} / \mathrm{min})$.

Anesthetic Influences: Anesthetic agents have a variable influence on $\mathrm{CBF}$ and $\mathrm{CMRO}_{2}$. (Table 1)

1. Inhalation anesthetics causes vasodilation but this vasodilatory effect is offset by metabolism mediated decrease in CBF. As a result, during low-dose 
inhalation anesthesia, CBF is either unchanged or slightly increased (sevoflurane in clinically relevant doses does not increase CBF but also associated with reduction in $\mathrm{CMRO}_{2}$.

2. Nitrous oxide causes vasodilatation of cerebral vessels and causes substantial increase in CBF and ICP when used alone in neurosurgeries. When $\mathrm{N}_{2} \mathrm{O}$ is used in combination with IV anesthetic agents and narcotics then the vasodilating property of $\mathrm{N}_{2} \mathrm{O}$ is completely inhibited and there is no increase in $\mathrm{CBF}$ and ICP. On the contrary, when $\mathrm{N}_{2} \mathrm{O}$ is used in combination with volatile anesthetic agents, then substantial increase in CBF and ICP is occurred.

3. Intravenous (IV) agents, including thiopental and propofol, cause vasoconstriction coupled with a reduction in $\mathrm{CMRO}_{2}$. Ketamine, on the other hand, increases CBF and metabolism.

4. Cerebral autoregulation, is abolished by inhalation agents in a dose-related manner but is preserved during propofol anesthesia (due to this propofol is induction agent of choice in neuro anesthesia)

Table 1: Effects of anesthetic agents on cerebral physiology

\begin{tabular}{|l|c|c|c|c|}
\hline \multicolumn{1}{|c|}{ Agents } & CMRO $_{2}$ & CBF & CBV & ICP \\
\hline Barbiturates & $\downarrow \downarrow \downarrow \downarrow$ & $\downarrow \downarrow \downarrow$ & $\downarrow \downarrow \downarrow \downarrow$ & $\downarrow \downarrow \downarrow$ \\
\hline Propofol & $\downarrow \downarrow \downarrow$ & $\downarrow \downarrow \downarrow$ & $\downarrow \downarrow$ & $\downarrow \downarrow$ \\
\hline Benzodiazepines & $\downarrow \downarrow$ & $\downarrow \downarrow$ & $\downarrow$ & $\downarrow$ \\
\hline Ketamine & $\uparrow$ & $\uparrow \uparrow \uparrow$ & $\uparrow \uparrow$ & $\uparrow \uparrow \uparrow$ \\
\hline Opioids & $\downarrow$ & $\downarrow$ & $\downarrow$ & $\downarrow$ \\
\hline N2O & $\downarrow$ & $\downarrow$ & $\downarrow$ & $\downarrow$ \\
\hline Halothane & $\downarrow \downarrow$ & $\uparrow \uparrow \uparrow \uparrow$ & $\uparrow \uparrow \uparrow$ & $\uparrow \uparrow \uparrow$ \\
\hline Isoflurane & $\downarrow \downarrow \downarrow$ & $\uparrow$ & $\uparrow$ & $\uparrow$ \\
\hline Sevoflurane & $\downarrow \downarrow \downarrow$ & $\uparrow$ & $\uparrow$ & $\uparrow \uparrow$ \\
\hline Desflurane & $\downarrow \downarrow \downarrow$ & $\uparrow$ & $\uparrow$ & $\uparrow \uparrow$ \\
\hline
\end{tabular}

$\mathrm{CMRO}_{2}$ : cerebral metabolic rate of oxygen; CBF: Cerebral blood flow

$\mathrm{CBV}$ : cerebral blood volume; ICP: Intracranial pressure

\section{CSF and Intracranial Pressure}

In a normal human the volume of CSF is about 150 $\mathrm{ml}$. It is continuously formed and absorbed with normal rate of production of CSF is about $500 \mathrm{ml} /$ day or 20 $\mathrm{ml} /$ hour or $0.3 \mathrm{ml} / \mathrm{minute}$. Around $60-70 \%$ of CSF is produced by the choroid plexus of ventricles, mainly the lateral. The remaining $30 \%$ is secreted directly from the ependymal cells lining the ventricles. The production of CSF is independent of ICP and everyday it is totally exchanged three times.

CSF in composition is essentially same as that of ECF of brain. Its formation involves active secretion of $\mathrm{Na}+$ from choroid plexuses along with water and is isotonic with plasma, though the concentration of $\mathrm{K}+$, bicarbonate and glucose is low. In comparison to plasma the protein content of CSF is very low. Normal CSF pressure which normally mirrors the ICP varies between 110 to $130 \mathrm{~mm}$ of $\mathrm{H}_{2} \mathrm{O}$. The CSF pressure rises on standing, coughing, sneezing and crying. Compressing of internal jugular vein increases the CSF pressure by inhibiting its flow in the intracranial venous sinuses (Queckenstedt's sign). Acetazolamide (carboxy anhydrase inhibitor), diuretics, corticosteroids, vasoconstrictor, etc, decrease the pressure of CSF by attenuating its secretion.

It is hypothesized that at any time the total volume of brain tissue, blood and CSF in cranial cavity will remain constant at a certain ICP which is known as the
Monro Kellie doctrin. Therefore, any increase in the volume of any one component must be offset by an equivalent decrease in volume of another component of cranial cavity to keep the ICP constant. As blood is the only compressible component of cranial cavity, so any increase or decrease of ICP the main burden will cause opposite change in cerebral blood volume. Increase in ICP during increase in intracranial volume by any reason is prevented naturally by four compensatory or buffering mechanisms:

1. Decrease in CSF production

2. Increase in CSF absorption

3. Displacement of CSF from cranial cavity to spinal compartment

4. Decrease in $\mathrm{CBF}$ and $\mathrm{CBV}$.

The major causes of increase in intracranial volume and increase in ICP are cerebral oedema due to any trauma, intracranial hemorrhage (extradural, subdural, subarachnoid) and intracranial mass. Increased ICP causes ischemia and nerve tissue damage in brain. The normal value of ICP at supine position is 10 to $15 \mathrm{~mm}$ of $\mathrm{Hg}$. If there is sustained elevation of ICP over $20 \mathrm{~mm}$ of $\mathrm{Hg}$ (usually pathological), then it is called intracranial hypertension. When ICP is high and CPP is low, interventions can target either ICP (maintain $<20 \mathrm{~mm}$ $\mathrm{Hg}$ ) or MAP to restore a favorable balance of the two. (Table-2) 
Table 2: Interventions for management of inadequate cerebral perfusion pressure

\begin{tabular}{|c|c|}
\hline Reduce brain water & $\begin{aligned} \text { i. } & \text { Mannitol } \\
\text { ii. } & \text { Hypertonic saline } \\
\text { iii. } & \text { Furosemide } \\
\text { iv. } & \text { Dexamethasone to decrease } \\
& \text { (peritumoral/vasogenic) edema }\end{aligned}$ \\
\hline $\begin{array}{l}\text { Remove CSF/ CSF } \\
\text { reduction }\end{array}$ & $\begin{array}{ll}\text { i. } & \text { External ventricular drain (EVD) } \\
\text { ii. } & \text { Lumbar drain } \\
\text { iii. } & \text { Acetazolamide (CSF reduction) }\end{array}$ \\
\hline $\begin{array}{l}\text { Decrease CBV/ } \\
\text { Decrease } \mathrm{CMRO}_{2}\end{array}$ & $\begin{aligned} & \text { i. } \text { Head up Tilt } \\
& \text { ii. Avoid constriction at the neck } \\
& \text { iii. Avoid PEEP and excessive pressure } \\
& \text { iv. Metabolic suppression: propofol, } \\
&\left.\text { barbiturate (decrease } \mathrm{CMRO}_{2}\right) \\
& \text { v. } \\
& \text { Mild to moderate hyperventilation }\end{aligned}$ \\
\hline $\begin{array}{l}\text { Elevate MAP }(\mathrm{CPP}= \\
\text { MAP -ICP })\end{array}$ & $\begin{array}{l}\text { i. Adequate intravascular volume } \\
\text { resuscitation } \\
\text { ii. Vasopressor }\end{array}$ \\
\hline
\end{tabular}

$\mathrm{CBV}=$ cerebral blood volume; $\mathrm{CSF}=$ cerebrospinal fluid; $\mathrm{MAP}=$ mean arterial pressure

Cerebral protection: Many medications and manipulations are used to maintain normal neurological parameters but best one includes mild to moderate hypothermia.

i. Profound hypothermia is well known for its neuroprotective effects. When core body temperature decreases below $20^{\circ} \mathrm{C}$, circulatory arrest of $<30$ minutes appears to be well tolerated.

ii. Mild hypothermia $\left(33^{\circ} \mathrm{C}-35^{\circ} \mathrm{C}\right)$ not only decreases cerebral metabolism but also modulates inflammatory response to ischemia, thus affecting the reperfusion portion of the injury as well.

iii. In the operating room during neurosurgical procedures, a temperature of $35^{\circ} \mathrm{C}$ to $36^{\circ} \mathrm{C}$ is reasonable.

iv. Volatile and IV anesthetic agents decrease cerebral metabolism.

v. Barbiturates and Propofol are used intraoperatively for short term decrease of cerebral metabolism.

vi. Hyperglycemia (>150 mg/dl) and cerebral ischemia in combination are harmful as hyperglycemia is associated with disruption of the blood brain barrier (BBB). This may promote cerebral edema and diffusion of excess calcium, lactate, and glutamate. So, tight glycemic control is a reasonable goal in these patients.

\section{E. Anesthesia for Neurosurgery}

\section{a) Preoperative Evaluation}

1. Patients for neurosurgery may present a wide range of signs and symptoms as an indication of raised ICP like headache, nausea and vomiting, hypertension and bradycardia, altered level of consciousness and sometimes papilledema. So a proper history and physical examination is must.
2. Preoperative risk stratification for a cardiac complication is important to consider. Current guidelines include delaying surgery:

i. for at least 2 weeks after simple balloon angioplasty,

ii. 4 to 6 weeks after placement of a bare metal stent, and

iii. 1 year after placement of a drug-eluting stent.

3. Many patients presenting for spine surgery have weakness or paralysis that may present a contraindication for succinylcholine (Sch).

4. Many neurosurgical patients are on antiepileptic medications. Previous allergies or reactions to these medications, especially phenytoin, should be elucidated.

5. A note should be made of antihypertensive drugs, drugs used for blood glucose control, and anticoagulants.

\section{b) Induction and Airway Management}

Induction and intubation are an art in neuro anesthesia for patients with elevated ICP and compromised cerebral compliance.

1. It is prudent to prevent the elevation of ICP at any cost by maintaining the arterial blood pressure at normal level and controlling ventilation to maintain $\mathrm{PaCO}_{2}$ between 30 and $35 \mathrm{mmHg}$.

2. At all the stages of induction and intubation procedure the hypoxia, hypercarbia and vasodilating agents should also be avoided at any cost.

3. For induction in neuro anesthesia the most commonly used agents are thiopentone, propofol and etomidate. Thiopentone and propofol not only reduce $\mathrm{CMRO}_{2}$ but also cause a significant reduction in $\mathrm{CBF}$ and ICP. 
4. During induction of anesthesia, three iatrogenic consequences (hypotension, hypertension, and apnea) may be significant hazards for neurosurgical patients.

i. Hypertension caused by laryngoscopy is poorly tolerated by patients after aneurysmal SAH because systolic hypertension is thought to be a cause of recurrent hemorrhage from the aneurysm.

ii. Hypertension may worsen elevated ICP and possibly lead to herniation of cranial contents into the foramen magnum.

iii. Apnea results in a predictable increase in $\mathrm{PaCO}_{2}$ and corresponding cerebral vasodilation.

1. A cervical collar for known or suspected cervical spine injury may make tracheal intubation more difficult. These patients are also particularly harmed by periods of hypotension or hypertension.

2. Because patients with SAH are at risk for harm from hypertension, it is reasonable to place an arterial catheter for hemodynamic monitoring before induction of anesthesia.

3. Many neurosurgical and spine surgery patients have conditions in which Sch is contraindicated.

4. In the setting of acute stroke or spinal cord injury (SCI), it remains safe to use Sch for approximately 48 hours from the time of injury. Succinylcholine may also increase ICP during fasciculation. So, it is not generally used in neuro anesthesia. But, succinylcholine is the agent of choice in neuro anesthesia when there is probability of difficult intubation or risk of aspiration.

5. Alternatively, a short-acting non depolarizing muscle relaxant is appropriate in many neurosurgical patients to achieve acceptable intubating conditions. Among these vecuronium, cis-atracuranium, rocuronium, etc. provide the greater hemodynamic stability.

6. The pressure response of laryngoscopy and intubation should be attenuated either by the use of any short acting opioids such as fentanyl in the dose of 2 to $3 \mu \mathrm{g} / \mathrm{Kg}$ or any of its congener just prior to induction and intubation. Other measures which attenuate this pressure response are the use of $\beta$-blockers (esmolol 0.5 to $1 \mu \mathrm{g} / \mathrm{Kg}$ ), or lignocaine $(0.5$ to $1 \mathrm{mg} / \mathrm{Kg} \mathrm{IV}$ $90 \mathrm{sec}$ before intubation) or deepening the anesthesia with the additional dose of thiopentone or propofol.

7. This intubation related pressure response can also be attenuated by hyperventilation with low dose (less than 1 MAC) of isoflurane and sevoflurane.
8. Vasodilators such as nitroprusside, calcium channel blockers, nitroglycerine, etc. should generally be avoided, till the dura is opened due to their potentially deleterious effect on $\mathrm{CBF}$ and ICP.

\section{c) Positioning}

Typically, the anesthesia provider will have limited access to the patient's head, so the endotracheal tube should be safely secured prior to draping. The breathing circuit, monitor cables, and intravenous and intra-arterial lines with suitable extension lines and accessible locations should be organized to avoid bulky tangling.

General considerations

i. Usually prolonged surgery

ii. Limited access to airway

iii. Careful identification of pressure areas

iv. Avoidance of traction on nerves

v. Thromboembolic precautions

1. Supine

i. Used for frontal, temporal or parietal access

ii. Extreme of head rotation may cause venous obstruction and sometimes carotid dissection

iii. Slight head-up usually desirable for venous drainage

iv. Slight flexion of elbows and knees is recommended

2. Semilateral

i. Used for retromastoid procedures

ii. Table tilted $10-20^{\circ}$, shoulder roll, head rotation

iii. Avoid extreme head rotation

\section{Lateral}

i. Used for posterior parietal and occipital access

ii. Axillary roll to prevent brachial plexus injury

iii. Stabilization with vacuum bean-bag or lateral rests (potential pressure areas)

4. Prone

i. Used for spinal, occipital, cranial suture and posterior fossa procedures

ii. For cervical spine and posterior fossa usually head-up and neck flexed

iii. Proper and careful planning for turning

iv. Secure airway and lines

v. Facial support much not cause eye compression and retinal ischaemia and edema

vi. Other pressure areas: elbows, breasts, iliac crests, genitalia, knees, toes should be well padded with cotton

vii. Avoid pressure on abdomen: increased PAW, IVC obstruction ( there must be enough space to move a hand below abdomen and table freely) 
viii. Neck flexion may cause compression of base of tongue and pharynx

ix. Endotracheal Tube must be well secured

5. Sitting

i. Used for some posterior fossa and cervical spine surgery, now a day's not a preferred position

ii. Possibly greater dangers of hypotension and cerebral ischaemia (decreased venous return, decreased CPP) than alternative positions

iii. Stockings or calf compression devices are must

iv. Tongue and pharynx compression or spinal injury from neck flexion

v. Pressure areas: buttocks, potential brachial plexus distraction must be well padded.

vi. Chances of Venous air embolism \pm paradoxical embolism are maximum ( $>25 \%$ incidence)

vii. Pneumocephalus may occur more frequently

a. May be worsened by $\mathrm{N}_{2} \mathrm{O}$ diffusion after dural closure (cease $\mathrm{N}_{2} \mathrm{O}$ with dural closure)

viii. Upper airway edema as a result of venous obstruction from excessive cervical flexion and quadriplegia from spinal cord compression and ischemia.

ix. An alternative approach is the "park bench position" in which the patient is placed in a lateral position but rolled slightly forward with the head further rotated to "look" at the floor (minimize the risk of air embolism)

6. Head Frame

i. Special consideration is given when a of a head frame using three pins (Mayfeld head clamp) is applied. Caution must be exercised to avoid bucking or movement during the placement and removal of the head frame and while the patient is fixed in the frame to avoid injury to the patient.

ii. Additional doses of propofol, or opioids, or both, are frequently administered right before the head frame placement to blunt the hemodynamic fluctuation.

iii. Local anesthesia injection at the site of pin insertion will also reduce the painful response to head clamp placement.

d) Venous air embolism (VAE) Though VAE occurs at any time during craniotomy in any position, but the peak incidence and occurrence time of it is during the sitting position (with open dural sinuses) and exposure of bony venous sinusoids at the time of dissection of bone and skin muscles. The factors which influence the severity of VAE are:

i. volume of air that enters the venous system,

ii. the rate of entry,

iii. the pressure of the right side of heart, iv. the presence or absence of patent foramen ovale, or VSD, and

v. Presence or absence of $\mathrm{N} 2 \mathrm{O}$.

1. The entry of large amount of air within a short period in venous system causes mechanical obstruction to right ventricular outflow and reflex pulmonary vasoconstriction with increased pulmonary arterial (PAP) pressure and decrease in the venous return to the left side of heart. Thus, cardiac output falls and cardiovascular collapse is resulted.

2. The entry of small amount of air in venous system has little clinical significant as it is absorbed at the pulmonary level only with little or no increase in PAP.

3. $\mathrm{N}_{2} \mathrm{O}$ if used during anaesthesia may increases the size of intravenous air bubble by 34 times because it is more soluble in blood than $\mathrm{N}_{2}$ and rapidly diffuses in the air bubbles. Hence, it is suggested that avoidance of $\mathrm{N}_{2} \mathrm{O}$ during neurosurgery enhances the safety, especially in sitting position.

4. Detection: The diagnosis of VAE is confrmed by blood gas analysis which shows increased difference in tension between $\mathrm{ETCO}_{2}$ and arterial $\mathrm{CO}_{2}$, in the absence of recent change in controlled ventilation. Hypoxaemia is the late feature of VAE. While an increase in intrapulmonary shunt leads to decline in $\mathrm{ETCO}_{2}$ excretion.

i. High sensitivity: TOE, precordial Doppler (right sternal edge 3rd-6th intercostal spaces). By Doppler method air bubbles as small as 0.5 $\mathrm{ml}$ can also be detected.

ii. Lower sensitivity: $\mathrm{ETCO}_{2}$, PAP

iii. Low sensitivity: indication of incipient arrest (BP, ECG, $\mathrm{SpO}_{2}$ )

1. Management

a. $\mathrm{ABC}$

b. Prevent further air entry

i. Notify surgeon, flood field with water

ii. Jugular compression, lower head

c. Manage intravascular air

i. $100 \% \mathrm{O}_{2}$, cease $\mathrm{N}_{2} \mathrm{O}$, cease PEEP

ii. Aspirate right heart: through CVP catheter, if present

d. Circulatory support: fluid, vasopressors, chest compression

e. Durant maneuver: Head-down- right lateral position (Not feasible in most neurosurgery)

\section{e) Monitoring during neuroanaesthesia}

Extensive monitoring during neuroanaesthesia is very important for better outcome, because neurosurgery is frequently complicated by rapid and 
large amount of blood loss. Different positions of patient required for surgery has different special complications, surgeries especially those in the posterior fossa may involve cardiovascular instability, hypotension, etc. So, all the patients undergoing neurosurgery need:

i. Pulse Oximeter

ii. A large bore intravenous cannula for rapid transfusion of crystalloid, colloid or blood.

iii. Arterial cannulation for beat to beat monitoring of arterial blood.

iv. Central venous cannulation for measurement of central venous pressure and diagnosis of VAE especially in high risk cases ( posterior fossa tumor / sitting position surgeries).

v. Capnography is used to adjust mechanical ventilation or assess spontaneous breathing.

vi. The electrocardiogram (ECG) allows prompt detection of cardiac dysrhythmias caused by surgical stimulation of brainstem or intracranial nerves.

vii. Neuromuscular blockade is monitored with a peripheral nerve stimulator.

\section{f) Maintenance of Anesthesia}

The basic considerations for maintenance of anesthesia include the type of monitoring planned for the procedure, brain relaxation, and the desired level of analgesia at the end of the surgical procedure.

1. During maintenance of neuroanaesthesia important factors to provide good quality of anaesthesia includes:

i. Maintenance of stable arterial BP (no hypertension, no hypotension),

ii. Avoidance of factors that lead to increased ICP (such as hypoxia, hypercarbia, vasodilating drugs, cerebral venous obstruction),

iii. Maintenances of adequate CPP, reduction of brain bulk (cerebral swelling), avoidance of cerebral ischaemia must be kept in mind.

iv. During the maintenance of neuro anaesthesia the airways, venous access and monitoring lines etc, should be well secured as the clinical monitoring is not possible intraoperatively due to inaccessibility of the upper trunk of patient.

v. Wrong ventilating patterns such as PEEP or rapid and small tidal volume ventilation should be avoided, if patient is not hypoxic. This is because they cause high mean airway pressure which has adverse effect on ICP by increasing CVP

2. Remifentanil is appropriate for neurosurgical procedures where tracheal extubation is planned at the end of the surgery and minimal residual sedation is desired to facilitate the neurologic examination.

3. Replacement of a volatile anesthetic with a continuous infusion of propofol is desirable with motor evoked potential (MEP) monitoring and when brain relaxation is inadequate with a volatile anesthetic.

4. The use of intraoperative muscle relaxants should be avoided during MEP, spontaneous electromyography, and cranial nerve monitoring. Muscle relaxants may be used during isolated somatosensory evoked potential monitoring.

\section{Ventilation pearls}

1. Hypocapnic cerebral vasoconstriction by hyperventilation helps in manipulating $\mathrm{CBF}$ and CBV. Hyperventilation is routinely used to provide brain relaxation and optimize surgical conditions.

2. During neurosurgical procedures, it is reasonable to maintain the $\mathrm{PaCO} 2$ between 30 and $35 \mathrm{~mm}$ $\mathrm{Hg}$. Further brain relaxation should be accomplished with other modalities, such as mannitol, hypertonic saline, or IV anesthesia.

3. If hyperventilation to a $\mathrm{PaCO} 2$ below $30 \mathrm{~mm} \mathrm{Hg}$ is required, it is appropriate to guide this therapy with jugular venous oximetry and the arterialjugular lactate gradient.

4. The duration of effectiveness of hyperventilation is limited. Normalization of $\mathrm{CBF}$ and consequently CBV occur within minutes. The beneficial effects of hyperventilation are more apparent in neurosurgical procedures of modest duration.

5. Isoflurane, Sevoflurane and Desflurane are used commonly in low doses ( $<1$ MAC) to control the intraoperative persistent hypertension, but at the cost of cerebral vasodilatation and increase the cerebral blood flow in different proportions. This effect is offset if slight hyperventilation is maintained at the same time. This is because increase in $\mathrm{CBF}$ caused by volatile anaesthetic agents is counteracted by the reduction of $\mathrm{CBF}$ caused by hyperventilation

6. The decrease in ICP by hyperventilation is more successful in isoflurane anesthesia than in sevoflurane and desflurane anesthesia.

\section{Fluid and electrolyte management}

1. To maintain adequate cerebral perfusion, adequate intravascular volume should be maintained (euvolemia to slight hypervolemia).

2. To minimize brain edema, it is important to maintain serum tonicity. It is prudent to check serum sodium levels on a regular basis in prolonged surgical procedures in which mannitol has been given.

3. Fluid restriction has no proven benefit but it is better than administration of hypotonic solution. It may also reduce hydrostatic pressure.

4. Hypertonic solutions reduce cerebral oedema. Hypertonic saline is as effective as mannitol or 
hypertonic colloids for reducing cerebral edema but has a disadvantage of hypernatremia, if not used judiciously.

5. The lower limit of acceptable hemoglobin or haematocrait has not been well defined. (Evidence supports avoidance of transfusion for a hematocrit above 21\%).Active hemodilution is not necessary and not of proven benefit.

6. Glucose Management: The combination of hyperglycemia and cerebral ischemia appears to be particularly deleterious. Nevertheless, tight glycemic control $(80-110 \mathrm{mg} / \mathrm{dL})$ with insulin may be associated with an increased mortality rate at 90 days. Because intensive insulin treatment results in increased variability in the blood glucose concentration, leading to cerebral osmotic shifts and higher incidences of hypoglycemia, leading to worse outcomes.

7. Dextrose solutions are not recommended because of the fear of cerebral edema due to movement of water form blood to brain. Crystalloid solutions such as normal saline, PlasmaLyte (multiple electrolytes injection) are preferred in the neurosurgical population.

\section{ICP reduction}

ICP reduction is a pressure concept used in the absence of craniotomy while brain relaxation is more a volume concept used during craniotomy. Various methods are used intraoperatively as per the case demand:

1. Mannitol (osmotic drug) in doses of 0.25 to $1 \mathrm{~g} / \mathrm{kg}$ IV is frequently used to reduce cerebral water content and decrease ICP before craniotomy, and to improve brain relaxation after craniotomy. The onset of action is 5 to 10 minutes, maximum effects are seen in 20 to 30 minutes, and its effects last for about 2 to 4 hours.

2. Mannitol should be given slowly because rapid infusion can also cause peripheral vasodilation (hypotension) and short-term intravascular volume expansion, which could result in increased ICP and intravascular volume overload.

3. Furosemide ( 0.5 to $1 \mathrm{mg} / \mathrm{kg}$ intravenously) is often used to decrease brain water and ICP and is likely synergistic with mannitol in decreasing ICP.

4. Intermittent intravenous injections of thiopental or propofol may also be effective in decreasing ICP.

5. When possible, the patient should be in a head-up position to avoid constriction around the neck, which may impair venous drainage.

\section{g) Extubation and Emergence}

The decisions that need to be made regarding emergence from anesthesia in neurosurgery patients depend mainly on the basis of the intactness of the neurological function.
1. Extubation if decided should be very smooth without coughing, bucking on endotracheal tube or any other type of straining which may precipitate the intracranial haemorrhage or worsen the cerebral oedema ( due to increased ICP).

2. Lignocaine (1 to $1.5 \mathrm{mg} / \mathrm{Kg} \mathrm{IV}$ ) or small dose of thiopentone (20 to $40 \mathrm{mg}$ ) or small dose of propofol (20 to $30 \mathrm{mg}$ ) 60-90 seconds before suctioning can be used, to suppress the coughing and bucking during extubation.

3. For extensive surgeries in the prone position, significant dependent edema frequently occurs. Combination of pronounced facial edema and an absent cuff leak after prone surgery should make one suspicious of upper airway edema. Delaying extubation of the trachea under these circumstances may be appropriate.

4. Postoperative pain after neurosurgery is less severe than the other form of surgeries. So, analgesics should be given very carefully and the overdose must be avoided so that neurological assessment of the patient can be done at appropriate time.

5. The postoperative stress response and resulting hyperdynamic events (hypertension, tachycardia) can be dealt by the judicious use of opioids and NSAIDS.

\section{References}

1. Handbook of Clinical Anesthesia $7^{\text {th }}$ Edition.

2. Textbook of anaesthesia for postgraduates, $1^{\text {st }}$ edition

3. Basics of anaesthesia, $6^{\text {th }}$ edition

4. Dinsmore J. Anaesthesia for elective neurosurgery. BJA, 2007 July; 99:1(1); 68-74.

5. Himmelseher S, Pfenninger E. Anaesthetic management of neurosurgical patients. Current Opinion in Anaesthesioogyl, 2001 Oct;14(5):483-90.

6. Campagna JA, Mi11er KW, Forman SA. Mechanisms of actions of inhaled anesthetics. N Engl J Med. 2003;348:2110-24.

7. Sutcliff AJ. Neuroanaesthesia: key points during the perioperative period.Minerva Anestesiol. 2003 Oct;69(10):739-44;

8. Umamaheswara Rao GS. What is New about Neuroanaesthesia? Indian J Anaesth. 2009 Aug;53(4):395-8.

9. Ramachandran R. Neuroanesthesia. Current Opinion in Anesthesiology: 2012 Oct;25 (5):p 513-515.

10. Pasternak JJ, Lanier WL. Neuroanesthesiology update. J Neurosurg Anesthesiol. 2014 Apr;26(2):109-54. 